\title{
Ganho de peso e produtividade animal em capim-marandu sob pastejo rotativo e adubação nitrogenada
}

\author{
Flávia Maria de Andrade Gimenes(1), Sila Carneiro da Silva ${ }^{(2)}$, Cleunice Auxiliadora Fialho(2), \\ Marcelo Barcelo Gomes ${ }^{(3)}$, Alexandre Berndt(1), Luciana Gerdes ${ }^{(1)}$ e Maria Tereza Colozza(1)
}

(1)Instituto de Zootecnia, Avenida Heitor Penteado, no 56, Centro, CEP 13460-000 Nova Odessa, SP. E-mail: flavia@iz.sp.gov.br, alberndt@iz.sp.gov.br, Igerdes@iz.sp.gov.br, colozza@iz.sp.gov.br (2)Universidade de São Paulo, Escola Superior de Agricultura Luiz de Queiroz, Avenida Pádua Dias, o 11, Vila Independência, CEP 13418-350 Piracicaba, SP. E-mail: scdsilva@esalq.usp.br, cleofialho@yahoo.com.br

(3)Universidade Federal de Goiás, Rua Riachuelo, № 1.530, Setor Samuel Graham, CEP 75804-020 Jataí, GO. E-mail: gomes_mb@yahoo.com.br

Resumo - O objetivo deste trabalho foi avaliar metas de manejo para capim-marandu (Urochloa brizantha cv. Marandu) submetido a pastejo rotativo e a doses de nitrogênio, de janeiro de 2009 a abril de 2010. Os tratamentos consistiram da combinação de duas frequências de pastejo (altura pré-pastejo de 25 e $35 \mathrm{~cm}$ ) e de duas doses de fertilizante nitrogenado (50 e $200 \mathrm{~kg} \mathrm{ha}^{-1}$ por ano) em delineamento de blocos ao acaso com arranjo fatorial $2 \times 2$ e quatro repetições. A altura de pós-pastejo estipulada foi de $15 \mathrm{~cm}$. Maiores valores de ganho de peso médio por animal por dia $\left(0,629\right.$ e $\left.0,511 \mathrm{~kg} \mathrm{dia}^{-1}\right)$ e por hectare $\left(886\right.$ e $\left.674 \mathrm{~kg} \mathrm{ha}^{-1}\right)$, bem como de taxa de lotação (3,13 e 2,85 UA ha-1), foram observados nos pastos manejados com altura pré-pastejo de $25 \mathrm{~cm}$. A aplicação de $200 \mathrm{~kg} \mathrm{ha}^{-1}$ de $\mathrm{N}$ resultou em aumentos na percentagem de folhas na massa de forragem pós-pastejo, nas taxas de acúmulo de forragem, na taxa de lotação e no ganho de peso por área. A mais adequada estratégia de manejo corresponde à altura pré-pastejo de $25 \mathrm{~cm}$, independentemente da dose de nitrogênio utilizada.

Termos para indexação: Urochloa brizantha, altura do dossel, altura pré-pastejo, altura pós-pastejo, manejo do pastejo, taxa de lotação.

\section{Weight gain and animal productivity on Marandu palisade grass under rotational stocking and nitrogen fertilization}

\begin{abstract}
The objective of this work was to assess management targets for marandu palisade grass (Urochloa brizantha $\mathrm{cv}$. Marandu) subjected to rotational stocking and to nitrogen fertilization rates, from January 2009 to April 2010. Treatments consisted of a combination of two grazing frequencies (pre-grazing heights of 25 and $35 \mathrm{~cm})$ and two levels of nitrogen application (50 and $200 \mathrm{~kg} \mathrm{ha}^{-1}$ per year). in a completely randomized block design, in a $2 \times 2$ factorial arrangement, with four replicates. The established post-grazing height was $15 \mathrm{~cm}$. Greater values of daily weight gain per animal per day $\left(0.629\right.$ and $\left.0.511 \mathrm{~kg} \mathrm{day}^{-1}\right)$, weight gain per hectare (886 and $674 \mathrm{~kg} \mathrm{ha}^{-1}$ ), and stocking rate (3.13 and $\left.2.85 \mathrm{AU} \mathrm{ha}^{-1}\right)$ were found for pre-grazing height of $25 \mathrm{~cm}$. The application of $200 \mathrm{~kg} \mathrm{ha}^{-1}$ of $\mathrm{N}$ increased the percentage of leaves in post-grazing forage mass, forage accumulation rate, stocking rate, as well as weight gain per hectare. The best grazing strategy corresponds to the pre-grazing height of $25 \mathrm{~cm}$, regardless of the nitrogen application.
\end{abstract}

Index terms: Urochloa brizantha, sward height, pre-grazing height, post-grazing height, grazing management, stocking rate.

\section{Introdução}

O avanço dos estudos de manejo do pastejo no Brasil permitiu ajustes mais refinados no planejamento e na execução de estratégias de manejo, as quais têm resultado em aumentos na produção de forragem e desempenho animal (Da Silva \& Carvalho, 2005). No entanto, a avaliação dessas estratégias em experimentos de pastejo, em grande escala, é escassa, uma vez que experimentos dessa natureza demandam grandes áreas e elevado número de animais, são difíceis de serem executados e têm custos elevados de condução (Difante et al, 2009, 2010).

Para o capim-marandu, Urochloa brizantha cv. Marandu (Syn. Brachiaria brizantha cv. Marandu), que ocupa a maior extensão de pastagens cultivadas do Brasil, não há conhecimento de experimentos avaliando desempenho animal de bovinos com o controle estrito de metas de estrutura dos pastos. Metas para o manejo do pastejo rotativo (Hodgson \& Da Silva, 2002) dessa forrageira correspondem à altura pré-pastejo de $25 \mathrm{~cm}$ (equivalente à condição em que ocorre interceptação 
de $95 \%$ da luz incidente pelo dossel forrageiro durante a rebrotação) e de pós-pastejo de $15 \mathrm{~cm}$ (Trindade et al. 2007; Giacomini et al., 2009a, 2009b). Essas metas foram determinadas em uma série de experimentos com escala reduzida e dose única de adubação nitrogenada. Embora o nitrogênio possa ser utilizado para acelerar o crescimento das braquiárias (Fagundes et al., 2005; Mesquita et al., 2010), há implicações quanto à redução do intervalo de pastejo e ao aumento na taxa de lotação necessários para a manutenção das metas de manejo e para o controle da estrutura do dossel forrageiro (Lemaire et al., 2009). Portanto, há necessidade de se avaliar a interação entre a fertilização nitrogenada e as metas de pastejo na tomada de decisões quanto ao manejo das pastagens.

O objetivo deste trabalho foi avaliar metas de manejo para capim-marandu (Urochloa brizantha cv. Marandu) submetido à pastejo rotativo e a doses de nitrogênio.

\section{Material e Métodos}

O experimento foi realizado no Instituto de Zootecnia de Nova Odessa, SP, pertencente à Agência Paulista de Tecnologia dos Agronegócios, em área de pastagem de capim-marandu, Urochloa brizantha (Hochst. ex A. Rich.) R.D. Webster cv. Marandu (Syn. Brachiaria brizantha cv. Marandu), de janeiro de 2009 a abril de 2010. As coordenadas geográficas aproximadas do local são $22^{\circ} 42^{\prime} \mathrm{S}$ e $47^{\circ} 18^{\prime} \mathrm{W}$, à $528 \mathrm{~m}$ de altitude. O solo é classificado como Latossolo Vermelho distrófico (Santos et al., 2006), com as seguintes características químicas, na camada de 0 a $20 \mathrm{~cm}$ : $\mathrm{pH}$ em $\mathrm{CaCl}_{2}$ de 4,5; $37,2 \mathrm{mg} \mathrm{dm}^{-3}$ de matéria orgânica; $1,6 \mathrm{mg} \mathrm{dm}^{-3}$ de fósforo; $14,9 \mathrm{mmol}_{\mathrm{c}} \mathrm{dm}^{-3}$ de cálcio; $10,4 \mathrm{mmol}_{\mathrm{c}} \mathrm{dm}^{-3}$ de magnésio; 2,1 $\mathrm{mmol}_{\mathrm{c}} \mathrm{dm}^{-3}$ de potássio; capacidade de troca de cátions de $68,1 \mathrm{mmol}_{\mathrm{c}} \mathrm{dm}^{-3}$; saturação por bases de $39,7 \%$. A interpretação da análise de solo foi feita de acordo com Werner et al. (1996). Os dados meteorológicos durante o período experimental foram coletados na Estação Meteorológica Automática, localizada a 4,0 km da área experimental (Figura 1).

A área experimental, de 48 ha, originalmente divididos em piquetes de 1,0 ha, foi preparada para o experimento por pastejos seguidos de roçadas de uniformização, com roçadora de arrasto, com altura de corte aproximada de $20 \mathrm{~cm}$, de agosto a dezembro de 2008. Esse procedimento foi necessário porque a área encontrava-se manejada de forma extensiva, com elevada massa de forragem, o que resultou em grande quantidade de material morto na base dos pastos durante o período experimental.

Após a uniformização das parcelas, iniciou-se o monitoramento das condições experimentais, tendo-se mensurado a altura do dossel forrageiro. A partir desse período, os pastos que atingiam a meta de altura prépastejo $(25$ ou $35 \mathrm{~cm}$ ) recebiam um lote de animais para pastejo até que a meta de altura pós-pastejo $(15 \mathrm{~cm})$ fosse atingida ou houvesse mudança de piquete. Os piquetes foram subdivididos por cerca elétrica em 96 unidades de 0,5 ha, para reduzir o período de ocupação de cada piquete e permitir maior flexibilidade de manejo em cada uma das unidades experimentais. A instalação da cerca elétrica foi iniciada em janeiro de 2009 e finalizada no início de março de 2009. O verão de 2009 foi considerado como período de adaptação dos pastos aos tratamentos de pastejo.

Os tratamentos consistiram de combinações entre dois intervalos de pastejo e duas doses de aplicação de nitrogênio $(\mathrm{N})$. Os intervalos de pastejo corresponderam ao período de tempo necessário para que o dossel forrageiro atingisse 25 e $35 \mathrm{~cm}$ de altura durante a rebrotação, isto é, 95\% e máxima interceptação da luz incidente (IL) pelo dossel forrageiro, respectivamente (Trindade et al., 2007; Giacomini et al., 2009a, 2009b). As doses de aplicação de $\mathrm{N}$ corresponderam a 50 e $200 \mathrm{~kg} \mathrm{ha}^{-1}$ por ano, consideradas suficientes para manutenção (Cadish et al., 1994; Werner et al., 1996) e produção (Fagundes et al., 2005; Oliveira et al., 2005), respectivamente. Os tratamentos foram alocados às unidades experimentais, em arranjo

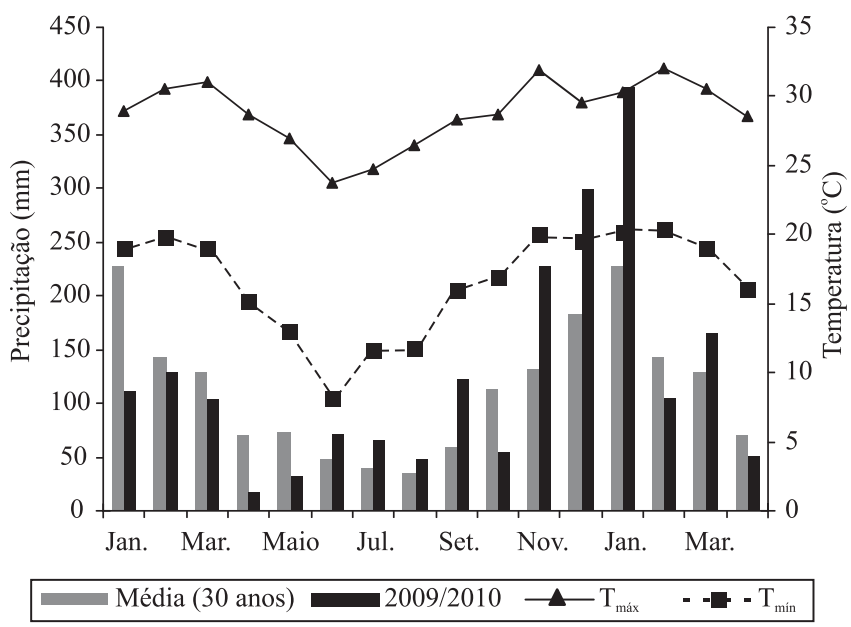

Figura 1. Precipitação e temperatura máxima e mínima mensais para o período de janeiro de 2009 a abril de 2010 e precipitação média mensal para o período de 1968 a 1990. 
fatorial $2 \times 2$, tendo-se utilizado delineamento de blocos completos ao acaso, com quatro repetições. Para todos os tratamentos, a meta de altura pós-pastejo (resíduo) foi de $15 \mathrm{~cm}$. Cada unidade experimental consistiu de um módulo de seis piquetes de 0,5 ha cada, o que totalizou 3,0 ha. Cada uma das 16 unidades experimentais recebeu três bovinos Nelore, com peso corporal médio inicial de $327 \mathrm{~kg}$, em 2009, e peso médio final de $521 \mathrm{~kg}$, em 2010, como animais-teste para mensuração do ganho de peso médio diário (GMD), além de um número variável de animais para ajustes na taxa de lotação, o que permitiu que as metas de manejo fossem executadas de acordo com as especificações dos tratamentos. Uma área reserva de 20 ha de capim-marandu foi utilizada para manutenção dos animais destinados ao ajuste de taxa de lotação.

A correção do solo foi feita com a aplicação de calcário dolomítico na dose de $1,0 \mathrm{Mg} \mathrm{ha}^{-1}$, em três blocos experimentais, e de $1,5 \mathrm{Mg} \mathrm{ha}^{-1}$ no bloco restante, de acordo com a meta de saturação por bases de 50\% (Werner et al., 1996). Foi aplicada adubação de NPK à lanço, com uso 25 e $100 \mathrm{~kg} \mathrm{ha}^{-1}$ de $\mathrm{P}_{2} \mathrm{O}_{5}$ e $\mathrm{K}_{2} \mathrm{O}$, respectivamente. Durante o primeiro verão, em janeiro de 2009 , foram realizadas adubações nitrogenadas à lanço $\left(50 \mathrm{~kg} \mathrm{ha}^{-1}\right)$, na forma de ureia ou nitrato de amônio, em todos os tratamentos. Em fevereiro e março de 2009, após a saída dos animais de cada piquete, foram realizadas mais duas aplicações parceladas $\left(75 \mathrm{~kg} \mathrm{ha}^{-1}\right)$ nos tratamentos com $200 \mathrm{~kg} \mathrm{ha}^{-1}$ de N. Na estação chuvosa de 2009/2010, as adubações nitrogenadas foram realizadas em novembro/dezembro (50 kg ha-1), e os $150 \mathrm{~kg} \mathrm{ha}^{-1}$ restantes para o tratamento de maior dose (200 $\left.\mathrm{kg} \mathrm{ha}^{-1}\right)$ em janeiro/fevereiro e março/ abril de 2010.

As condições experimentais foram monitoradas por meio de avaliações periódicas da altura do dossel forrageiro, com um bastão medidor (Barthram, 1985), ao longo de linhas transectas que cobriam toda a área de cada um dos 96 piquetes, em pré e pós-pastejo (100 leituras por piquete). A meta de altura pós-pastejo de $15 \mathrm{~cm}$ foi utilizada como valor de referência para indicar a menor altura de resíduo permitida, pois foi necessário acelerar a rotação dos piquetes em resposta ao aumento das taxas de acúmulo de forragem e à não disponibilidade de número suficiente de animais para o ajuste necessário na taxa de lotação. Assim, foi priorizado o ajuste na taxa de lotação sempre que houve número suficiente de animais. Quando não, a alternativa foi variar a duração do período de ocupação dos pastos para assegurar que a meta pré-pastejo fosse mantida, tendo-se permitido variação de $5 \%$.
O acompanhamento do manejo em cada unidade experimental foi realizado semanalmente, tendo-se considerado a condição do piquete em pastejo e dos dois piquetes a serem pastejados, na sequência. $\mathrm{O}$ manejo dos animais dentro de cada unidade experimental foi feito com base no acompanhamento do crescimento e da proximidade da meta de 25 ou $35 \mathrm{~cm}$ de altura pré-pastejo, conforme o tratamento. Durante a rebrotação, o monitoramento visual da altura dos pastos foi realizado diariamente. Era feita uma previsão do número de dias até que o próximo piquete da sequência estivesse em condições de receber os animais. Essa previsão foi utilizada para definir o período de ocupação do piquete em uso e assegurar que o término do pastejo em um piquete coincidisse com o início no próximo e assim sucessivamente.

Para as avaliações agronômicas das plantas, foi escolhido um piquete de amostragem por unidade experimental que representasse o conjunto de piquetes que compunham cada uma das unidades experimentais (módulos de seis piquetes). A interceptação de luz pelo dossel forrageiro foi mensurada na condição pré-pastejo, nos 16 piquetes avaliados, com uso de aparelho analisador de dossel marca LI-COR, modelo LAI 2000 (LI-COR, Lincoln, Nebraska, EUA), cujo funcionamento foi descrito por Welles \& Norman (1991). Foram utilizadas seis estações de leitura (amostragem) por piquete. Em cada estação, eram realizadas uma leitura acima do dossel e cinco no nível do solo, o que totalizou 6 pontos de leitura acima do dossel e 30 pontos de leitura no nível do solo, por piquete. As avaliações de interceptação de luz foram feitas após a estabilização dos pastos aos tratamentos de pastejo, no final da primavera de 2009 e do verão de 2010, para avaliar se as metas de altura pré-pastejo correspondiam às condições planejadas de $95 \%$ e máxima IL.

A cada ciclo de pastejo, amostras de forragem foram coletadas nas condições de pré e pós-pastejo nos piquetes de amostragem por meio do corte da forragem contida no interior de seis armações metálicas de $0,25 \times 2,00 \mathrm{~m}$ por piquete. O corte foi realizado no nível do solo em pontos representativos da condição média dos pastos. Cada amostra foi subdividida em duas subamostras, uma para determinação do teor de matéria seca (MS) e outra para determinação da composição botânica/morfológica da forragem. As amostras destinadas à determinação do teor de MS foram secas em estufa de circulação forçada de ar a $65^{\circ} \mathrm{C}$, até peso constante. Já as amostras submetidas à separação manual, tiveram seus componentes botânicos 
(capim-marandu e outras espécies) e morfológicos (lâminas foliares, bainhas foliares + colmos, e material morto) separados e submetidos à secagem em estufa, de forma análoga às amostras para determinação do teor de MS. A massa de forragem foi calculada com base na massa de MS dos componentes morfológicos, enquanto a taxa de acúmulo de forragem foi obtida apenas com o material vivo (folhas+colmos), pois, conforme mencionado, havia elevada quantidade de material morto nos pastos.

Foram realizadas avaliações de perdas por pastejo $\left(\mathrm{kg} \mathrm{ha}{ }^{-1}\right.$ ), de acordo com a metodologia descrita por Carnevalli et al. (2006), e coletadas amostras de simulação de pastejo pelo método "hand-plucking" (Sollenberger \& Cherney, 1995), nas fases inicial e final do pastejo, imediatamente após a entrada e antes da saída dos animais dos piquetes. As amostras foram submetidas à separação morfológica e, após secagem e moagem, enviadas para determinação de proteína bruta (PB) (Helrich, 1990), digestibilidade in vitro da matéria seca (DIVMS), fibra em detergente neutro (FDN), fibra em detergente ácido (FDA), lignina (Goering \& Van Soest, 1970) e matéria mineral. As avaliações de ganho de peso foram realizadas pela pesagem dos animais a cada 28 dias, após jejum de água e alimento de 14 horas, tendo-se utilizado os pesos para ajustes em taxa de lotação e cálculos de ganho de peso por unidade de área.

Os intervalos variáveis de pastejo para cada tratamento e repetição podem ser atribuídos à forma como os tratamentos foram definidos e à condução independente de cada unidade experimental. Embora representem mudanças potencialmente importantes no padrão de resposta ao longo do período experimental, os resultados de caracterização dos pastos e dos regimes de pastejo (janeiro de 2009 a abril de 2010) foram agrupados em épocas do ano, dentro das quais o comportamento das variáveis estudadas era relativamente uniforme: verão de 2009 (fevereiro a março de 2009), outono de 2009 (abril a junho de 2009), inverno de 2009 (julho a setembro de 2009), primavera de 2009 (outubro a dezembro de 2009) e verão de 2010 (janeiro a abril de 2010).

A análise de variância dos dados foi realizada com uso do Proc Mixed do SAS (SAS Institute, 2003). Utilizou-se o critério de informação de Akaike para escolha da matriz de covariância (Wolfinger, 1993). Os efeitos de altura pré-pastejo, dose de $\mathrm{N}$ e épocas do ano e suas interações foram considerados fixos, enquanto o efeito de blocos foi considerado aleatório (Littel et al., 2000). As médias dos tratamentos foram estimadas pelo teste LSMeans e comparadas, quando necessário, pela probabilidade da diferença (PDIFF), com o teste t de Student, a 5\% de probabilidade. Para análise dos dados de número total de pastejos e ganho de peso total, foi utilizado o procedimento GLM do SAS, a 5\% de probabilidade.

\section{Resultados e Discussão}

Aaltura média do dossel forrageiro em prée pós-pastejo, para os tratamentos de 25 e $35 \mathrm{~cm}$, foi de 25,2 e $34,9 \mathrm{~cm}$ e de 16,2 e 20,4 cm, respectivamente (Figura 2A). No verão
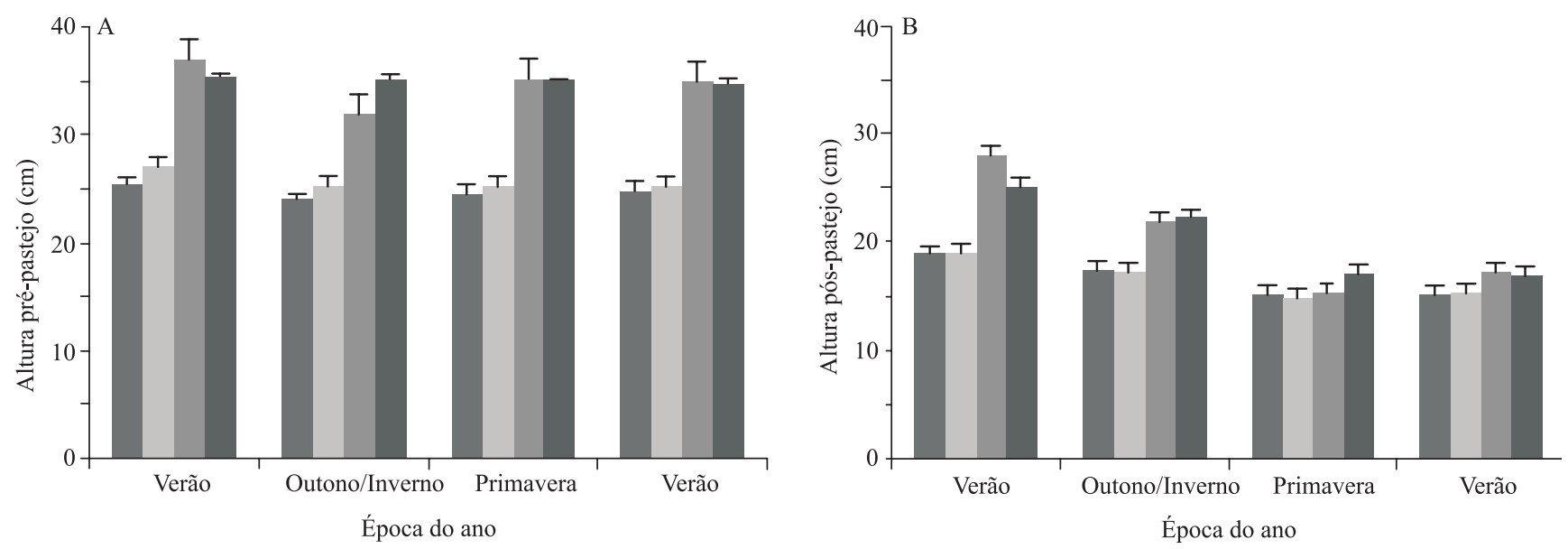

$\llbracket 25 / 50 \backsim 25 / 200 \backsim 35 / 50 \backsim 35 / 200$

Figura 2. Altura pré-pastejo (A) e pós-pastejo (B) de pastos de capim-marandu submetidos às alturas de pré-pastejo de 25 e $35 \mathrm{~cm}$ e às doses de adubação nitrogenada de 50 e $200 \mathrm{~kg} \mathrm{ha}^{-1}$, de fevereiro de 2009 a abril de 2010. 
de 2009, as metas de altura pré-pastejo estiveram próximas das metas propostas; no entanto, ficaram abaixo da meta, no outono/inverno de 2009, para o tratamento $35 \mathrm{~cm} / 50 \mathrm{~kg} \mathrm{ha}^{-1}(31,9 \mathrm{~cm})$, e na primavera de 2009 , para o tratamento $25 \mathrm{~cm} / 50 \mathrm{~kg} \mathrm{ha}^{-1}(21,0 \mathrm{~cm})$. Esse resultado pode ser explicado pelas baixas taxas de acúmulo de forragem nos pastos adubados com $50 \mathrm{~kg} \mathrm{ha}^{-1}$ de $\mathrm{N}$ durante o período frio e seco do ano (Tabela 1) e pela necessidade de se manterem três animais-teste em cada unidade experimental, o que causou interrupção do pastejo antes das alturas pós-pastejo serem atingidas. Como as plantas entram em estádio reprodutivo no final do verão e no início do outono, não foi possível rebaixar os piquetes até $15 \mathrm{~cm}$, o que resultou na retirada dos animais dos pastos com resíduos mais altos. No verão e no outono/inverno de 2009, as alturas pós-pastejo estiveram acima da meta (Figura 2 B), pois não havia animais suficientes para os ajustes necessários na taxa de lotação. Na primavera de 2009 e no verão de 2010, período de crescimento vegetativo intenso das plantas, as alturas pós-pastejo dos tratamentos $35 \mathrm{~cm} / 50 \mathrm{~kg} \mathrm{ha}^{-1}$ e $35 \mathrm{~cm} / 200 \mathrm{~kg} \mathrm{ha}^{-1}$ foram superiores a $15 \mathrm{~cm}$, o que pode ser atribuído ao acúmulo de colmos vegetativos, principalmente nos tratamentos com $200 \mathrm{~kg} \mathrm{ha}^{-1}$ de N. Durante o período de rebrotação, depois que o pasto atinge 95\% de interceptação luminosa, há menor acúmulo de folhas e maior acúmulo de colmos e de material

Tabela 1. Taxas de acúmulo de forragem e de lotação em pastos de capim-marandu submetidos a diferentes doses de adubação nitrogenada ${ }^{(1)}$.

\begin{tabular}{|c|c|c|c|}
\hline \multirow[t]{2}{*}{ Época } & \multicolumn{2}{|c|}{ Dose de nitrogênio $\left(\mathrm{kg} \mathrm{ha}^{-1}\right.$ de $\left.\mathrm{N}\right)$} & \multirow[t]{2}{*}{ Média } \\
\hline & 50 & 200 & \\
\hline & \multicolumn{3}{|c|}{ Acúmulo de forragem $\left(\mathrm{kg} \mathrm{ha}^{-1}\right.$ por dia) } \\
\hline Verão de 2009 & $14,1 \pm 12,19 \mathrm{Bb}$ & $103,0 \pm 13,11 \mathrm{Aa}$ & $58,5 \pm 8,94 \mathrm{~A}$ \\
\hline Outono de 2009 & $10,3 \pm 11,28 \mathrm{Ba}$ & $23,2 \pm 11,28 \mathrm{Ca}$ & $16,8 \pm 7,97 \mathrm{~B}$ \\
\hline Inverno de 2009 & $17,0 \pm 11,28 \mathrm{Ba}$ & $4,3 \pm 11,28 \mathrm{Ca}$ & $10,7 \pm 7,97 \mathrm{~B}$ \\
\hline Primavera de 2009 & $48,5 \pm 11,28 \mathrm{Aa}$ & $67,3 \pm 11,28 \mathrm{Ba}$ & $57,9 \pm 7,97 \mathrm{~A}$ \\
\hline Verão de 2010 & $55,7 \pm 12,19 \mathrm{Aa}$ & $61,9 \pm 11,28 \mathrm{Ba}$ & $58,8 \pm 8,30 \mathrm{~A}$ \\
\hline \multirow[t]{2}{*}{ Média } & $29,1 \pm 5,63 b$ & $51,9 \pm 5,64 a$ & \\
\hline & \multicolumn{3}{|c|}{ Taxa de lotação (UA ha'-1) } \\
\hline Verão de 2009 & $2,70 \pm 0,15 \mathrm{Ba}$ & $2,78 \pm 0,14 \mathrm{Ca}$ & $2,74 \pm 0,10 \mathrm{~B}$ \\
\hline Outono de 2009 & $2,03 \pm 0,14 \mathrm{Cb}$ & $3,13 \pm 0,15 \mathrm{Ba}$ & $2,58 \pm 0,10 \mathrm{~B}$ \\
\hline Inverno de 2009 & $1,12 \pm 0,14 \mathrm{Db}$ & $1,60 \pm 0,14 \mathrm{Da}$ & $1,36 \pm 0,10 \mathrm{C}$ \\
\hline Primavera de 2009 & $3,32 \pm 0,14 \mathrm{Ab}$ & $5,09 \pm 0,15 \mathrm{Aa}$ & $4,20 \pm 0,10 \mathrm{~A}$ \\
\hline Verão de 2010 & $3,59 \pm 0,14 \mathrm{Ab}$ & $4,59 \pm 0,15 \mathrm{Aa}$ & $4,09 \pm 0,10 \mathrm{~A}$ \\
\hline Média & $2,55 \pm 0,05 b$ & $3,44 \pm 0,05 \mathrm{a}$ & \\
\hline
\end{tabular}

morto (Carnevalli et al., 2006; Pedreira et al., 2007; Da Silva et al., 2009), o que contribui para aumentar as dificuldades de pastejo e reduz o valor nutritivo da forragem produzida (Da Silva \& Carvalho, 2005; Trindade et al., 2007).

A massa e o acúmulo de forragem foram calculados com base na massa de MS de folhas e colmos, em decorrência da elevada percentagem de material morto nos pastos, principalmente no verão e no outono/ inverno de 2009. Pastos manejados com a altura de pré-pastejo de $35 \mathrm{~cm}$ apresentaram maiores valores de massa de forragem pré-pastejo $\left(6.680 \mathrm{~kg} \mathrm{ha}^{-1} \mathrm{de}\right.$ MS) do que pastos manejados a $25 \mathrm{~cm}\left(4.800 \mathrm{~kg} \mathrm{ha}^{-1}\right.$ de MS). Maiores valores de massa de forragem foram registrados no outono de $2009\left(6.590 \mathrm{~kg} \mathrm{ha}^{-1}\right.$ de MS), enquanto os menores foram observados no verão, no inverno e na primavera do mesmo ano (5.270 $\left.\mathrm{kg} \mathrm{ha}^{-1} \mathrm{de} \mathrm{MS}\right)$.

Com exceção do inverno de 2009 , as variações em massa de forragem pós-pastejo foram relativamente pequenas durante o ano; contudo, valores maiores foram registrados no verão e no outono de 2009 (3.940 $\mathrm{kg} \mathrm{ha}^{-1}$ de MS), em comparação à primavera de 2009 e ao verão de 2010 (2.900 kg ha-1 de MS), resultado consistente com as alturas pós-pastejo superiores a $15 \mathrm{~cm}$ registradas para essas épocas do ano (Figura $2 \mathrm{~B}$ ). Nos pastos manejados a $35 \mathrm{~cm}$ de altura pré-pastejo, a aplicação de $200 \mathrm{~kg} \mathrm{ha}^{-1}$ de $\mathrm{N}$ resultou em aumento da massa de forragem pós-pastejo (de 3.530 para $4.490 \mathrm{~kg} \mathrm{ha}^{-1}$ de MS), o que não ocorreu nos pastos manejados com altura pré-pastejo de $25 \mathrm{~cm}$ (3.310 $\mathrm{kg} \mathrm{ha}^{-1}$ de MS). As alturas pré-pastejo de 25 e $35 \mathrm{~cm}$ corresponderam aos valores de IL de 94,6 e $97,4 \%$, respectivamente, independentemente da dose de $\mathrm{N}$ utilizada, o que confirma os dados obtidos por Trindade et al. (2007) e Giacomini et al. (2009a, 2009b), em experimento realizado em piquetes de área reduzida, tendo-se considerado a interceptação luminosa como variável controle e a altura pré-pastejo como variável resposta. Esses resultados indicam o potencial do uso da altura do pasto como variável prática de manejo em condições de campo, para facilitar o planejamento, o monitoramento e o controle do processo de pastejo.

As alterações no intervalo entre pastejos (Figura $3 \mathrm{~A}$ ) podem ser explicadas pelas mudanças no período de ocupação dos piquetes (Figura $3 \mathrm{~B}$ ), pois as decisões quanto aos ajustes na taxa de lotação foram feitas com base no tempo que os animais 
poderiam permanecer no piquete atual antes que o próximo atingisse a altura pré-pastejo especificada como meta. A aplicação de $\mathrm{N}$ acelerou o crescimento das plantas, o que reduziu o período de ocupação do piquete e, consequentemente, aumentou o número de ciclos de pastejo, com o maior número registrado para o tratamento $25 \mathrm{~cm} / 200 \mathrm{~kg} \mathrm{ha}^{-1}(9,3$ ciclos) e o menor para o tratamento $35 \mathrm{~cm} / 50 \mathrm{~kg} \mathrm{ha}^{-1}$ (5,1 ciclos). As mudanças na taxa de lotação e no período de ocupação foram feitas de acordo com a sazonalidade do acúmulo de forragem (Tabela 1), particularmente quando o número de animais não foi suficiente para a realização dos ajustes necessários.

A taxa de lotação foi superior nos pastos manejados a $25 \mathrm{~cm}$ de altura pré-pastejo, em comparação aos manejados a $35 \mathrm{~cm}: 3,13$ e 2,85 $\mathrm{UA} \mathrm{ha}^{-1}$, respectivamente. A taxa de lotação variou com a estação do ano, e a dose de $200 \mathrm{~kg} \mathrm{ha}^{-1}$ de $\mathrm{N}$ resultou em maior taxa de lotação em todas as estações, exceto no verão de 2009 (Tabela 1). O peso corporal dos animais aumentou consistentemente durante o período experimental (Figura 4), e o ganho de peso diário foi maior nas unidades experimentais manejadas com altura pré-pastejo de $25 \mathrm{~cm}$ (Figura 5), exceto no verão e na primavera de 2009. No verão de 2009, as alturas pós-pastejo estiveram acima da meta, para todos os tratamentos, em virtude do número insuficiente de animais para ajuste na taxa de acúmulo de forragem. Para tornar a rotação das unidades experimentais
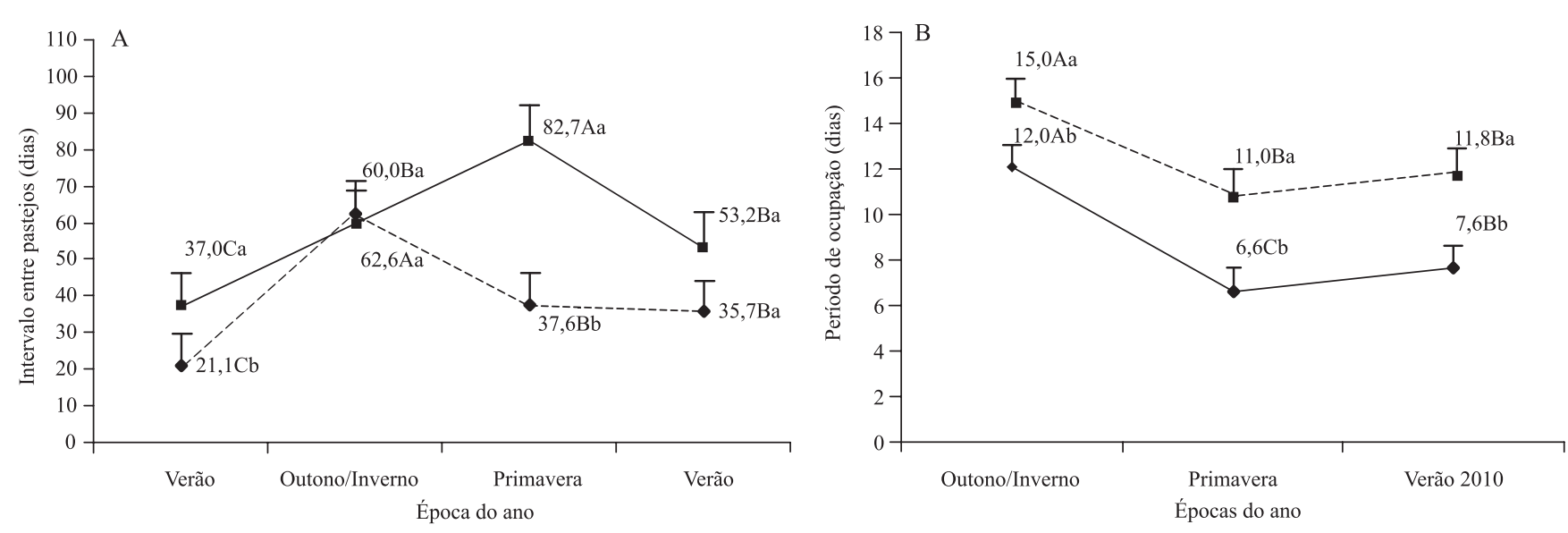

Figura 3. Intervalo entre pastejos, de fevereiro de 2009 a abril de 2010 (A), e período de ocupação, de abril de 2009 a abril de 2010 (B), de pastos de capim-marandu submetidos a pastejo rotativo com alturas pré-pastejo de 25 e $35 \mathrm{~cm}$. Médias seguidas de letras iguais, minúsculas entre alturas pré-pastejo e maiúsculas entre épocas do ano, não diferem entre si pelo teste t, a 5\% de probabilidade.

Pesq. agropec. bras., Brasília, v.46, n.7, p.751-759, jul. 2011

mais rápida e cumprir a meta pré-pastejo, o resíduo pós-pastejo foi mantido acima de $15 \mathrm{~cm}$, condição que favoreceu o desempenho animal, principalmente nos pastos de $35 \mathrm{~cm}$ pré-pastejo. Os animais não foram forçados a pastejar o estrato mais baixo do dossel, em que colmos velhos e material morto estavam concentrados, o que reduziu o contraste entre os tratamentos.

$\mathrm{Na}$ primavera de 2009, as chuvas que restauram os níveis de água no solo iniciaram-se apenas em novembro, o que obrigou os animais a ocupar os piquetes por mais tempo e a pastejar os resíduos no estrato mais baixo, próximo à meta de altura pós-pastejo, com redução nos valores de massa pós-pastejo. Essa condição restringiu o consumo para todos os tratamentos, o que resultou em menor desempenho animal neste período (Figura 5). No verão de 2010, os valores de GMD atingiram praticamente a metade dos registrados no verão de 2009 (Figura 5), o que pode ser explicado pela quantidade excessiva de chuvas em 2010 (Figura 1), pela menor altura dos pastos em pós-pastejo (Figura 2 B), e pelo uso de animais-teste com peso corporal médio diferente no verão de 2009 (peso inicial de $327 \mathrm{~kg}$ ) e de 2010 (peso final de $521 \mathrm{~kg}$ ), com padrão de deposição de tecidos (músculo vs. gordura) e eficiência de conversão alimentar distintos (Oltjen et al., 1988).

O melhor desempenho dos animais nos pastos manejados a $25 \mathrm{~cm}$ foi influenciado pelo melhor 
valor nutritivo da forragem consumida, caracterizada pela maior percentagem de folhas $(83,1$ vs $77,3 \%)$ e pela menor percentagem de colmos $(9,3$ vs $11,9 \%)$ e de material morto (7,2 vs 11,2\%), nas amostras de simulação de pastejo. Esses resultados estão de acordo com os obtidos por Trindade et al. (2007), em experimento análogo, em escala de piquete. Assim, pastos de capim-marandu com altura pré-pastejo de

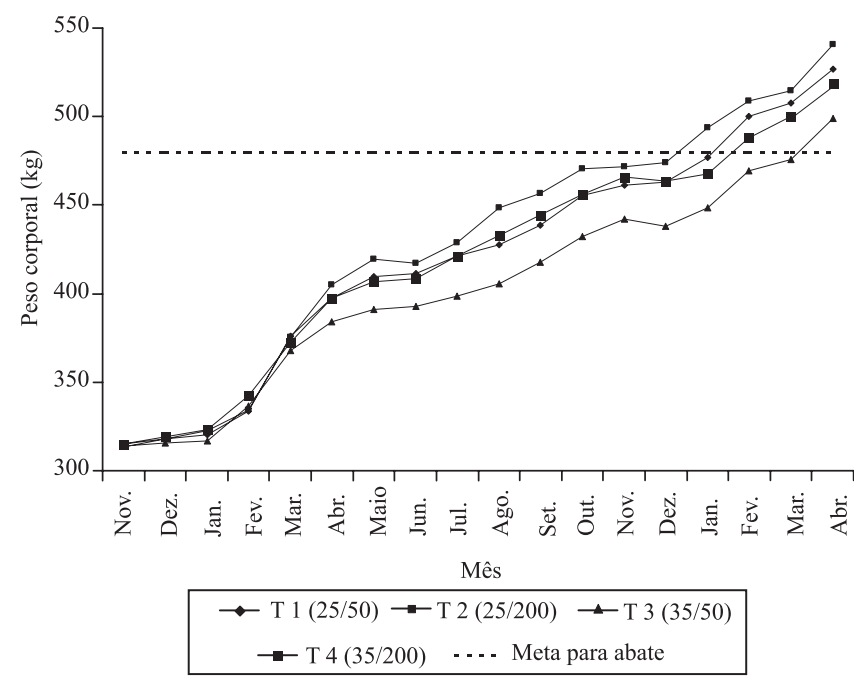

Figura 4. Evolução mensal do peso corporal de novilhos Nelore em pastos de capim-marandu submetidos a estratégias de pastejo rotativo e à adubação nitrogenada, de fevereiro de 2009 a abril de 2010.

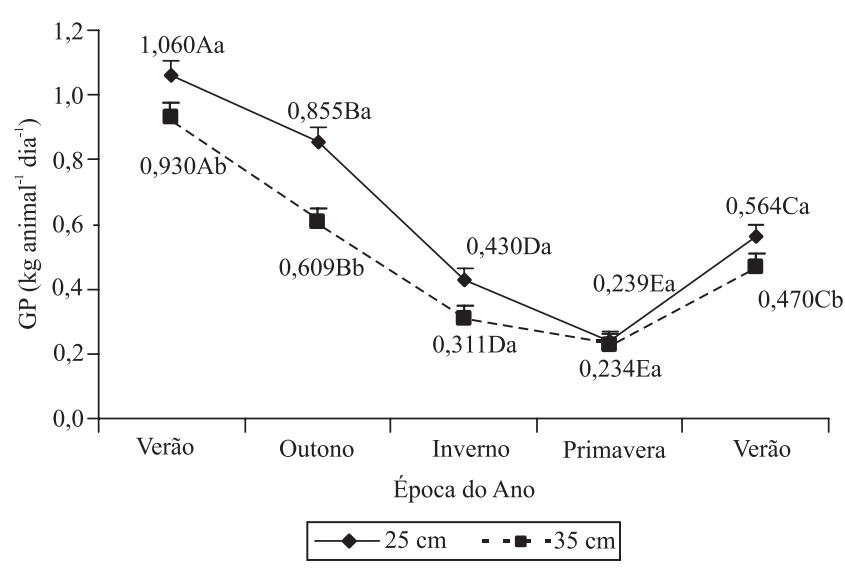

Figura 5. Médias de ganho de peso (GP) médio diário de bovinos Nelore em pastos de capim-marandu submetidos a alturas de pré-pastejo de 25 e $35 \mathrm{~cm}$, de fevereiro de 2009 a abril de 2010. Letras minúsculas comparam efeito de altura pré-pastejo dentro das épocas do ano, e maiúsculas comparam efeito de época do ano dentro das alturas pré-pastejo.
$25 \mathrm{~cm}$ rebaixados por bovinos até a altura de resíduo de $15 \mathrm{~cm}$ apresentam maior percentagem de folhas e menor percentagem de colmos e de material morto na extrusa, particularmente no final do período de pastejo. No presente trabalho, obteve-se maior valor nutritivo, caracterizado por maiores valores de $\mathrm{PB}$ e digestibilidade, e menores valores de FDN e FDA, nos pastos manejados com o tratamento $25 \mathrm{~cm} / 200 \mathrm{~kg} \mathrm{ha}^{-1}$. Quanto à $\mathrm{PB}$, não houve diferença significativa entre as alturas de 25 e $35 \mathrm{~cm}$ (9,40 e 9,41\%, respectivamente), na dose de $50 \mathrm{~kg} \mathrm{ha}^{-1}$ de N. No entanto, na dose de $200 \mathrm{~kg} \mathrm{ha}^{-1}$, pastos manejados a $25 \mathrm{~cm}$ apresentaram teor de PB $(11,80 \%)$ superior aos manejados a $35 \mathrm{~cm}$ $(10,36 \%)$.

Pastos manejados a $25 \mathrm{~cm}$ de altura pré-pastejo apresentaram DIVMS superior $(65,79 \%)$ aos manejados a $35 \mathrm{~cm}(64,03 \%)$. A dose de $200 \mathrm{~kg} \mathrm{ha}^{-1}$ de N contribuiu para o aumento da DIVMS de 63,28 para $66,54 \%$. Para a altura pré-pastejo de $25 \mathrm{~cm}$, o maior teor de FDN ocorreu na dose de $50 \mathrm{~kg} \mathrm{ha}^{-1}$ de $\mathrm{N}(64,47 \%)$, em comparação com a de $200 \mathrm{~kg} \mathrm{ha}^{-1}(61,72 \%)$. Não houve diferença em FDN na altura de $35 \mathrm{~cm}$, para as doses de $\mathrm{N}$ avaliadas $(66,49 \%)$. Pastos manejados a $35 \mathrm{~cm}$ apresentaram maior teor de FDN que pastos manejados a $25 \mathrm{~cm}$, independentemente da dose de $\mathrm{N}$ utilizada. $\mathrm{O}$ teor de FDA foi maior na altura pré-pastejo de $35 \mathrm{~cm}(34,28 \%)$ do que na de $25 \mathrm{~cm}(32,11 \%)$. A dose de $50 \mathrm{~kg} \mathrm{ha}^{-1}$ de $\mathrm{N}$ apresentou maior teor de FDA $(33,41 \%)$, em comparação à dose de $200 \mathrm{~kg} \mathrm{ha}^{-1}$ de $\mathrm{N}(32,99 \%)$.

Ao final do experimento, o ganho de peso total por unidade de área foi maior nos pastos manejados a $25 \mathrm{~cm}\left(886 \mathrm{~kg} \mathrm{ha}^{-1}\right)$ do que nos manejados a $35 \mathrm{~cm}\left(674 \mathrm{~kg} \mathrm{ha}^{-1}\right)$. Dessa forma, a deficiência das atuais recomendações de uso e manejo do pastejo do capim-marandu no Brasil fica evidente, uma vez que alturas de entrada de $45 \mathrm{~cm}$ (Correa, 2000) e $60 \mathrm{~cm}$ (Pereira, 2003) são normalmente preconizadas. O uso de alturas pré-pastejo superiores a $25 \mathrm{~cm}$ pode elevar as perdas por pastejo, que foram maiores nos pastos com altura pré-pastejo de $35 \mathrm{~cm}$, em comparação aos de $25 \mathrm{~cm}$ (20,3 e 24,1\% da massa de forragem pré-pastejo, respectivamente). Em valores absolutos, o ganho de peso por unidade de área nos pastos manejados com altura pré-pastejo de 25 e $35 \mathrm{~cm}$ passou de 770 para $1.010 \mathrm{~kg} \mathrm{ha}^{-1}$ e de 630 para $720 \mathrm{~kg} \mathrm{ha}^{-1}$ com o aumento da dose de $\mathrm{N}$ de 50 para $200 \mathrm{~kg} \mathrm{ha}^{-1}$, respectivamente. Não houve diferença significativa em ganho de peso 
entre os tratamentos $25 \mathrm{~cm} / 50 \mathrm{~kg} \mathrm{ha}^{-1}\left(770 \mathrm{~kg} \mathrm{ha}^{-1}\right)$ e $35 \mathrm{~cm} / 200 \mathrm{~kg} \mathrm{ha}^{-1}$ (720 kg ha-1) (Figura 4), embora tenha-se utilizado uma quantidade quatro vezes maior de fertilizante.

\section{Conclusões}

1. A estratégia de manejo mais adequada para o capim-marandu em pastejo rotativo corresponde às alturas de pré-pastejo de $25 \mathrm{~cm}$, independentemente da dose de nitrogênio utilizada.

2. O aproveitamento eficiente da forragem pelo manejo adequado do pasto é prioritário sobre o aumento de produção de forragem pela aplicação de fertilizantes nitrogenados.

\section{Agradecimentos}

À Fundação de Amparo à Pesquisa do Estado de São Paulo, pelo apoio financeiro; ao Dr. Joaquim Carlos Werner e ao Dr. João José Assumpção de Abreu Demarchi, pela colaboração na elaboração e na condução do experimento.

\section{Referências}

BARTHRAM, G.T. Experimental techniques: the HFRO sward stick. In: The Hill Farming Research Organization. Biennial report. Midlothian: HFRO, 1985. p.29-30.

CADISH, G.; SCHUNKE, R.M.; GILER, K.E. Nitrogen cycling in a pure grass pasture and a grass-legume mixture on a red latosol in Brazil. Tropical Grasslands, v.28, p.43-52, 1994.

CARNEVALLI, R.A.; DA SILVA, S.C.; BUENO, A.A.O.; UEBELE, M.C.; BUENO, F.O.; HODGSON, J.; SILVA, G.N.; MORAES, J.P.G. de. Herbage production and grazing losses in Panicum maximum cv. Mombaça under four grazing managements. Tropical Grasslands, v.40, p.165-176, 2006.

CORRÊA, L. de A. Produção de carne em pastagens adubadas. São Carlos: Embrapa Pecuária Sudeste, 2000. 25p. (Embrapa Pecuária Sudeste. Circular técnica, 25).

DA SILVA, S.C.; BUENO, A.A. de O.; CARNEVALLI, R.A.; UEBELE, M.C.; BUENO, F.O.; HODGSON, J.; MATTHEW, C.; ARNOLD, G.C.; MORAES, J.P.G. de. Sward structural characteristics and herbage accumulation of Panicum maximum cv. Mombaça subjected to rotational stocking management. Scientia Agricola, v.66, p.8-19, 2009.

DA SILVA, S.C.; CARVALHO, P.C.F. de. Foraging behaviour and intake in the favourable tropics/sub-tropics. In: MCGILLOWAY, D.A. (Ed.). Grassland: a global resource. Wageningen: Academic, 2005. p.81-95.

DIFANTE, G. do S.; EUCLIDES, V.P.B.; NASCIMENTO JÚNIOR, D. do; DA SILVA, S.C.; BARBOSA, R.A.; TORRES JÚNIOR,
R.A. de A. Desempenho e conversão alimentar de novilhos de corte em capim-tanzânia submetido a duas intensidades de pastejo sob lotação rotativa. Revista Brasileira de Zootecnia, v.39, p.33-41, 2010.

DIFANTE, G. dos S.; EUCLIDES, V.P.B.; NASCIMENTO JÚNIOR, D. do; DA SILVA, S.C.; TORRES JÚNIOR, R.A. de A.; SARMENTO, D.O. de L. Ingestive behavior, herbage intake and grazing efficiency of beef cattle steers on Tanzania guineagrass subjected to rotational stocking managements. Revista Brasileira de Zootecnia, v.38, p.1001-1008, 2009.

FAGUNDES, F.L.; FONSECA, D.M. da; GOMIDE, J.A.; NASCIMENTO JÚNIOR, D. do; VITOR, C.M.T.; MORAIS, R.V. de; MISTURA, C.; REIS, G. da C.; MARTUSCELLO, J.A. Acúmulo de forragem em pastos de Brachiaria decumbens adubados com nitrogênio. Pesquisa Agropecuária Brasileira, v.40, p.397-403, 2005.

GIACOMINI, A.A.; DA SILVA, S.C.; SARMENTO, D.O. de L.; ZEFERINO, C.V.; SOUZA JÚNIOR, S.J.; TRINDADE, J.K. da; GUARDA, V. del'A.; NASCIMENTO JÚNIOR, D. do. Growth of marandu palisadegrass subjected to strategies of intermittent stocking. Scientia Agricola, v.66, p.733-741, 2009a.

GIACOMINI, A.A.; DA SILVA, S.C.; SARMENTO, D.O. de L.; ZEFERINO, C.V.; TRINDADE, J.K. da; SOUZA JÚNIOR, S.J.; GUARDA, V. del'A.; SBRISSIA, A.F.; NASCIMENTO JÚNIOR, D. do. Components of the leaf area index of marandu palisadegrass swards subjected to strategies of intermittent stocking. Scientia Agricola, v.66, p.721-732, 2009b.

GOERING, H.K.; VAN SOEST, P.J. Forage fiber analysis (apparatus, reagents, procedures, and some applications). Washington: U.S. Agriculture Research Service, 1970. 379p. (Agricultural handbook, 379).

HELRICH, K. Official methods of analysis of the Association of Official Analytical Chemists. 15.ed. Arlington: AOAC, 1990. 2v., 1298p.

HODGSON, J.; DA SILVA, S.C. Options in tropical pasture management. In: REUNIÃO ANUAL DA SOCIEDADE BRASILEIRA DE ZOOTECNIA, 39., 2002, Recife. A produção animal e a sociedade brasileira: anais. Recife: Sociedade Brasileira de Zootecnia, 2002. p.180-202.

LEMAIRE, G.; DA SILVA, S.C.; AGNUSDEI, M.; WADE, M.; HODGSON, J. Interactions between life lifespan and defoliation frequency in temperate and tropical pastures: a review. Grass and Forage Science, v.6, p.341-352, 2009.

LITTEL, R.C.; PENDERGAST, J.; NATARAJAN, R. Modelling covariance structure in the analysis of repeated measures data. Statistics in Medicine, v.19, p.1793-1819, 2000.

MESQUITA, P. de; DA SILVA, S.C.; PAIVA, A.J.; CAMINHA, F.O.; PEREIRA, L.E.T.; GUARDA, V. del'A.; NASCIMENTO JÚNIOR, D. do. Structural characteristics of marandu palisadegrass swards subjected to continuous stocking and contrasting rhythms of growth. Scientia Agricola, v.67, p.23-30, 2010.

OLIVEIRA, P.P.A.; TRIVELIN, P.C.O.; OLIVEIRA, W.S. de; CORSI, M. Fertilização com $\mathrm{N}$ e $\mathrm{S}$ na recuperação de pastagem de Brachiaria brizantha cv. Marandu em Neossolo Quartzarênico. Revista Brasileira de Zootecnia, v.34, p.1121-1129, 2005. 
OLTJEN, J.W.; GARRETT, W.N. Effects of body weight, frame size and rate of gain on the composition of gain of beef steers. Journal of Animal Science, v.66, p.1732-1748, 1988.

PEDREIRA, B.C. e; PEDREIRA, C.G.S.; DA SILVA, S.C. Estrutura do dossel e acúmulo de forragem de Brachiaria brizantha cultivar Xaraés em resposta a estratégias de pastejo. Pesquisa Agropecuária Brasileira, v.42, p.281-287, 2007.

PEREIRA, J.C. Manejo de pastagens. Brasília: Senar, 2003. 92p. (Senar. Trabalhador na bovinocultura de leite. Coleção, 72).

SANTOS, H.G. dos; JACOMINE, P.K.T.; ANJOS, L.H.C. dos; OLIVEIRA, V.A. de; OLIVEIRA, J.B. de; COELHO, M.R.; LUMBREAS, J.F.; CUNHA, T.J.F. (Ed.). Sistema brasileiro de classificação dos solos. 2.ed. Rio de Janeiro: Embrapa Solos, 2006. $306 \mathrm{p}$.

SAS INSTITUTE. Statistical analysis system: user's guide. Versão 9.1. Cary: SAS Institute, 2003.

SOLLENBERGER, L.E.; CHERNEY, D.J.R. Evaluating forage production and quality. In: HEATH, M.E.; BARNES, R.F.;
METCALFE, D.S. (Ed.). Forages: the science of grassland agriculture. Ames: Iowa State University, 1995. p.97-110.

TRINDADE, J.K. da; DA SILVA, S.C.; SOUZA JÚNIOR, S.J. de; GIACOMINI, A.A.; ZEFERINO, C.V.; GUARDA, V. del'A.; CARVALHO, P.C.F. de. Composição morfológica da forragem consumida por bovinos de corte durante o rebaixamento do capim-marandu submetido a estratégias de pastejo rotativo. Pesquisa Agropecuária Brasileira, v.42, p.883-890, 2007.

WELLES, J.M.; NORMAN, J.M. Instrument for indirect measurement of canopy architecture. Agronomy Journal, v.83, p.818-825, 1991.

WERNER, J.C.; PAULINO, V.T.; CANTARELLA, H. Forrageiras. In: RAIJ, B. van; CANTARELLA, H.; QUAGGIO, J.A.; FURLANI, A.M.C. (Ed.). Recomendações de adubação e calagem para o Estado de São Paulo. 2.ed. Campinas: Instituto Agronômico, 1996. p.263-273. (IAC. Boletim técnico, 100).

WOLFINGER, R. Covariance structure selection in general mixed models. Communications in Statistics - Simulation and Computation, v.22, p.1079-1106, 1993.

Recebido em 20 de julho de 2010 e aprovado em 3 de maio de 2011 